\title{
Supporting Gut Health by Homeostasis and Intrinsic Mechanisms
}

\section{Jennifer Gantzer*}

Department of Clinical Sciences, National University of Health Sciences, USA

*Corresponding Author: Jennifer Gantzer, Department of Clinical Sciences, National University of Health Sciences, USA.
Received: September 27, 2021

Published: October 6, 2021

(C) All rights are reserved by Jennifer Gantzer.

\begin{abstract}
Optimal digestion serves many physiological roles including assimilation of nutrients, sustaining the commensal bacterial environment of the gut microbiome, supporting daily bowel movements and elimination of toxins. An individual's health and quality of life can be sustained and/or lost at the interface of the external world entering the gut as gut-brain, nervous system, and microbial forces all interact to influence gut function and digestion. This review of digestive function and loss thereof focuses on ways to optimize gut health by mimicking the intrinsic homeostasis of the Rest and Digest parasympathetic regulation of secretions and motility and the bidirectional gut-brain cross-talk of the microbial commensals. Supporting mastication, digestive secretions, motility, and the microbiome can significantly restore gastrointestinal homeostasis and its vital role in overall health.
\end{abstract}

Keywords: Digestion; Assimilation; Dysbiosis; Microbiome; Gut-brain Axis; Natural Therapy

\section{Introduction}

Optimal digestion serves many physiological roles including assimilation of nutrients, sustaining the commensal bacterial environment of the gut microbiome, supporting daily bowel movements and elimination of toxins. An individual's health and quality of life can be sustained and/or lost at the interface of the external world entering the gut as gut-brain, nervous system, and microbial forces all interact to influence gut function and digestion. This review of digestive function and loss thereof focuses on ways to optimize gut health by mimicking the intrinsic homeostasis of the Rest and Digest parasympathetic regulation of secretions and motility and the bidirectional gut-brain cross-talk of the microbial commensals. Supporting mastication, digestive secretions, motility, and the microbiome can significantly restore gastrointestinal homeostasis and its vital role in overall health.

\section{Symbiotic microbiota}

The gut is an intricate microbial ecosystem with different micro-environments in the small bowel compared to that of the large intestine and colon. It has been well-established the microbiome needs to exist in harmony and serves symbiotic functions including vitamin and micronutrient synthesis, neurotransmitter formation and its correlated role in mood/depression/anxiety, epigenetic regulation, protection from mucosal injury and heavy metal toxicity, and digestive motility optimal functioning and bowel transit times [1-7]. Miro-Blanch and Yanes explain, "Hosts and their microbiota have a very intimate relationship and should be considered as a single biological and evolutionary unit, termed holobiont" and "however, unbalanced microbiota can also cause disease" [4].

To experience these beneficial symbiotic features of our gut microbiome we must exist in a balance of species and their population 
distributions; when the balance is lost, the subsequent changes in bacterial species and/or bacterial populations then aberrantly altered bacterial metabolic generated byproducts induce irregular bowel movements, digestive discomfort, systemic and constitutional symptoms, and with chronic imbalances disease onset [410]. Much of this influence is a joint effort of eating habits and food selection [11].

\section{Symbiotic co-habitation - eating habits and food selection}

Establishment of symbiotic co-habitation with our gut microbes requires simple lifestyle habits that are easily over-looked [12-14]. Dietary choices, lifestyle and eating habits, and the ability to both digest and absorb our food are at the top of the list for optimal gut microbiomes [1,14].

\section{Eating habits}

The simple act of sitting, enjoying, and chewing our food is the most basic of principles and so easily forgotten. After chewing we must achieve assimilation of food nutrients as the combination of digestion and absorption.

First, support the parasympathetic "Rest and Digest" autonomic nervous system mechanism that influences digestive juices and digestive motility by designating time for only eating. This means sit and eat; take the time to enjoy the food. This supports the digestive process of enzymatic secretions at all levels starting with saliva, the gastric enzyme and gastric acid secretions, down through pancreatic exocrine enzyme and bicarbonate secretions, gallbladder bile fat emulsifying secretions, and continues to include all the gut neurotransmitter signals to the smooth muscles for gut motility and/ or opening/closing of sphincters $[15,16]$.

In addition, the pre-meal cephalic phase is the initial "rampup" of stimulatory neurotransmitter synapses on the glands and smooth muscle preparing them for an incoming meal, as well as routing increased blood to the visceral organs of the abdomen for digestion, secretions, absorption, motility, and optimal bowel habits. This is achieved by utilizing food prep and the senses such as touching, smelling, feeling, and seeing the food as well as the cognitive appreciation of the meal and its flavors and textures. These simple food prep steps immensely prepare the body for successful digestion and should be paired with the enjoyment of sitting, eating, and chewing food [1,15-17].
Next is chewing; a lot of chewing. Chewing not only mixes the food bolus with entry level digestive enzymes but also starts the degradative process of food matrix separation for further down gastric and intestinal release of essential vitamins/minerals, essential amino acids and fatty acids that must be broken apart from their dietary structures. Efficient chewing increases the surface area to enhance food particle mixing with gastric hydrochloric acid juices and proteolytic enzymes for protein degradation. An undigested, mal-chewed food "piece" floating in the stomach is like Mount Everest to a molecule of digestive enzyme [15,18].

In addition to the necessary act of chewing, is the vital importance of low stomach acid and the need for hydrochloric acid. Mealtime low stomach acid is multi-purpose acting as a potent antimicrobial for pathogenic microbes entering the body contaminated in food, for stimulating gastric digestive enzymes/secretions, and directly assisting in the food particle degradative process. The acidity of chyme exiting the stomach entering the gastro-intestinal junction at the duodenum then also directly stimulates further digestive secretions from gallbladder and pancreas. Furthermore, bile secretions, like gastric acid, also plays a dual role in digestion and protection. Bile is a potent antimicrobial further guarding against pathogens entering the intestinal tract from contaminated food and/or drink as a second line of defense if not killed by acidic gastric juices, while also increasing the surface area for fat digestion lipid emulsification [19-22].

Without the synergistic actions of parasympathetic "Rest and Digest" coordinated by chewing and followed by digestive secretions then food particles entering the intestinal lumen remain incompletely digested unable to be optimally absorbed by the intestinal brush border cells and become excess nutrient for gut microbes promoting overgrowth and shifts in gut bacterial populations.

\section{Food selection}

Neurotransmitter signaling for digestive secretions and motility along with properly functioning digestive processes play a vital role in health through nutrient assimilation and for sustaining balanced species/populations through the intestines and colon. However, that alone is not sufficient for complete sustainability of symbiosis.

Just as eating diversity of food selections to ensure ingestion of all the "essential" food categories our body cannot synthesize 
on its own such as the essential - vitamins, minerals, amino acids, fatty acids $[23,24]$ and dietary antioxidant food choices for protection against oxidative stress, our food selections also significantly impact and influence the species and population counts of the gut microbiome lending towards symbiosis or taking us down a path of digestive despair [25].

Microbiome diversity is the key to symbiosis because of its role in creating the optimal microenvironment of species with different metabolic requirements generating different metabolic byproducts; anaerobes, facultative anaerobes, hydrogen-producers, hydrogen-consumers, methane-producers, methane-consumers [26]. The average healthy human possesses 1014 different bacteria in their gut with upwards of $70 \%$ of the gut microbiome consisting of Eubacterium, Bifidobacterium, Lactobaccili, Bacteroides/ Prevotella, and Firmicutes including Clostridium/Eubacterium as the primary players with more than $90 \%$ being Bacteroides and Firmicutes and over $50 \%$ being 75 bacterial species within these phyla shared by healthy individuals [26-28].

Nightly cortisol levels negatively influence microbiome life cycle with down regulation in population count and die off as well as lack of incoming nutrients required for their own metabolism further lowering quantities of microbial populations. Therefore, upon rising and before the first meal, the gut microbiome exists at low counts with the nascent populations remaining from the day before, until after the first meal when their numbers significantly rise upon nutrient entry into the ileocecal region promoting bacterial growth and fermentation of insoluble food products powering their revival [28].

The hierarchy of species and the need for their diversity is demonstrated in the daily routine as the nascent populations await nutrient substrate to begin reproduction and life cycle. The daily requirement for dietary fibers begins here. Dietary fibers are nonabsorbable starches and grains which remain in the gut lumen to feed the microbiome. In turn they metabolize these as indigestible carbs generating the necessary gaseous byproducts which then feed and saturate the distal intestine and colonic cells. In healthy individuals, host-microbiome harmony occurs when the species/ populations of gut microbes co-exist in robust and balanced quantifiable colony-forming units (CFUs); as a collective they generate nutrients such as amino acids and vitamins, they offer non-threat- ening immune surveillance, and metabolic breakdown products such as short chain fatty acids (SCFA) and anaerobic byproduct intestinal gases that feed the enterocytes and colonocytes. The latter generation of the SCFAs acetate, propionate, and butyrate, as well as the intestinal gases hydrogen, methane, and hydrogen sulfide perfuse the focal tissues acting as an energy source for the underlying epithelial and lamina propria cells as well as function to support and enhance the protective gut barrier by decreasing susceptibility to mucosal injury [27,30].

The generation of colonic anaerobic Hydrogen Sulfide (H2S) maintains the strength and integrity of the mucus layer and intestinal barrier [31], increases resistance to antibiotics, protects from free radical damage from inflammatory reactive oxygen species [32], and attenuates the detrimental gastric effects of NSAIDinducing enteropathy drugs [30] All of these actions rely on daily ingested dietary fiber entering the upper small bowel as nutrient substrate and energy sources for "high abundance" bacterial species to hydrolyze insoluble sugars which act as nutrient substrates and energy sources for the distal small bowel and colonic commensal bacterial anaerobe populations $[1,12,14]$.

Additionally, through the bidirectional communications of the gut-brain access, the presence of the commensal species in addition to the local responses of the neuroendocrine cells to the commensal bacterial metabolic by-products generates a crosstalk of the central nervous system and the gut, the Gut-Brain Axis, and is an interplay of the brain's effect on gut barrier, permeability, secretions, motility, and activity of the GALT and gut immune system sustaining balance and optimal gut/digestive function. All of the above benefits become harmful and symptomatic when they are produced excessively as seen in dysbiosis.

\section{Dysbiosis and SIBO}

Dysbiosis is a species/population shift outside the quantifiable ranges established from metabolomic studies and typically present with changes in bowel movements as well as a host of non-digestive systemic extra-intestinal symptoms ranging from headaches [33], skin abnormalities [34], mood changes [35], brain fog [36], insomnia [37], fatigue [38], and many more subtle constitutional symptoms [20]. Dysbiosis is a disturbing loss of symbiosis with our gut microbes. Their imbalances favor a pathophysiological 
platform of inflammation and aberrant gas production instead of healthy protective roles $[20,28]$. Similarly, increased Firmicutes/ Bacteroides ratio have been observed in obesity, hypertension, IBS, and autism [28].

Gut dysbiosis in its simplest form is imbalanced species and populations of the gut microbiome. Dysbiosis can occur solely in the species/populations of the colon and distal small bowel where majority of the microbiome exists, but it can also occur when the colonic microbiome begin to populate more proximally upwards into the small bowel.

Small intestinal bacterial overgrowth (SIBO) is defined as an increase in the concentration of bacteria of more than 100,000 colony-forming units (CFUs) per mL of proximal jejunal fluid. The occurrence of SIBO is not necessarily opportunistic or pathogenic bacterial infections but simply combinations of aerobic and anaerobic bacteria of the large intestine which have populated proximally in excessive numbers into the small bowel. SIBO can also commonly occur with SIFO, small bowel fungal overgrowth. The primary bacterial overgrown colonies in SIBO are Streptococcus, Enterococcus, Klebsiella, and E. coli whereas the fungal overgrowth in SIFO is Candida [11,20,39].

Dysbiosis and the progression towards SIBO is very common in the population of chronic hypochlorhydria patients on long-term PPIs attributed to raising the gastric juice $\mathrm{pH}$ with its inhibitory effects on digestion and antimicrobial activity; chronic use of PPIs is associated with increased enteric infections in addition to its causal onset of dysbiosis. A 2017 health symposium determined chronic use of PPIs with their negative impact gastric stomach acid $\mathrm{pH}$ to be more detrimental to the microbiome than the use of antibiotics. "Analysis of the gut microbiome revealed that the use of proton pump inhibitors (PPIs) was associated with a significant decrease in gut microbiota diversity and with significant changes of around $20 \%$ of bacterial taxa. This adverse effect of PPIs on bacterial diversity was greater than for any other drug class, including antibiotics" $[40,41]$. In addition to chronic use of antacids after eating meals and/or chronic use of PPIs each inducing a history of hypochlorhydria, it is important to also rule out an $H$. Pylori infection which induces gastric hypochlorhydria $[42,43]$.

Recall dietary choices and eating habits also significantly influence onset or avoidance of dysbiosis, SIBO, SIFO occurrence rates.
Digestive disturbances and dysbiosis onset will occur when any of the following occur: poor dietary choices especially food diversity and lack of daily dietary fiber, poor eating habits including inefficient designated sit/eat time, inadequate chewing, and chronic hypochlorhydria. Once these microbiome aberrant shifts occur the predominant issue becomes the onset of Irritable Bowel Syndrome (IBS).

Dysbiosis, SIBO, and hypochlorhydria are IBS instigators. SIBO is very common in IBS and resolving SIBO removes symptoms of IBS. Lin explains nearly all symptoms of IBS are wholly consistent with SIBO including: $[44,45]$

- Postprandial bloating (nearly universal symptom)

- Physical evidence of small bowel gas irrespective of predominant symptoms

- High prevalence of abnormal lactulose breath test results

- Dramatic reduction in symptoms when antimicrobial therapy is given ( $\mathrm{Rx}$ and natural)

- $\quad$ Altered gut motility

- Visceral hypersensitivity

- Abnormal brain-gut interactions

- Evidence of autonomic dysfunction

- Nearly uniform immune activation regardless of prior acute gastroenteritis

- Extraintestinal symptoms often flu-like in quality.

\section{Gut barrier intestinal permeability}

Dysbiosis and SIBO are aberrant shifts in the gut microenvironment based upon changes in the bacterial species and their concordant populations with overgrowth of some species and lack thereof other species tending a loss of diversity, the hallmark of gut-bug symbiosis and gut function. These aberrant microbial shifts present with a host of symptoms and disease presentation. Loss of the protective intestinal gut barrier presents with an increased pathogenicity from the microbiome aberrant contact with enterocytes and its GALT thereby allowing immune cell activation to food particles, potentially contaminated strains of non-commensal bacteria, and/or commensal bacteria in the setting of dysbiosis/SIBO each setting up a potential towards development of food sensitivities and/or an over activated immune system. There is an additional risk of translocation of the bacterial species and their endotoxemic 
pro-inflammatory lipopolysaccharide outer layer (LPS) into circulation which is also associated with a wide array of insidious onset of symptoms and disease.

The integrity of the intestinal barrier is just as vital in health and disease prevention and/or reversal and is determined by a properly functioning gut barrier interface. The intestinal barrier consists of a sterile mucus layer abutting the lamina propria of the tissue with the necessary biofilm of commensal microbiome populations sandwiched in the middle and the intestinal lumen on the exterior side facing the passing fecal matter $[30,40]$. It is sophisticated and under tight control as a thin barrier intentionally designed for close proximity to luminal nutrients however the close proximity also increases vulnerability to exposure to pathogenic microbes and toxins. The properly functioning tight junctions of the intestinal barrier allow water and few electrolytes through like a series of ziplock closures. This ziplock structural feature exists as tiny holes near the luminal surface created by proteins which act as Velcro on one side (zonula occludens and claudins) which connect to the opposite side (myosin light chain kinase) forming a physiological pore which opens upon kinase activity thereby opening the tight junction $[1,46]$. The microbial biofilm layer under normal conditions remains separate from the abutting mucus layer which undergoes morphological geographical change as it thickens further towards the large intestine away from the single layer in the small intestine [1]. Breach of the gut barrier and loss of the sterile mucus layer sets the stage for leaky gut and translocation of whole bacterial species into the systemic circulation. The onset of leaky gut is loss of the gut barrier integrity which poses the body with the burden of commensal bacteria and their immune stimulating LPS cross-over from the gut lumen into the circulation inducing low-grade endotoxemia and/or direct liver damage $[21,47]$. Many causal agents of leaky gut syndrome correlate back to diet and lifestyle habits and removal of these habits with incorporation of better and purposeful choices improves and corrects the loss of gut barrier. Chronic ingestion of gluten through the zonulin/gliadin mechanism [48,49]. and chronic unmanaged stress are two of the top-rated inducers of leaky gut [50] followed by microbial dysbiosis [51] which correlates back to diet, lifestyle, and eating habits.

\section{Returning to symbiosis and sustaining gut function homeosta-} sis

The onset and diagnosis of dysbiosis, SIBO/SIFO, and leaky gut are reversible and even preventable. They coexist as underlying drivers of digestive and constitutional diseases and they are extinct in the symbiotic homeostatic healthy individual and population who do not exist with daily digestive distress and onslaught of chronic diseases.

When dysbiosis, SIBO/SIFO, and/or leaky are suspected, then testing is the best place to start to confirm the extent of gut overgrowth and leaky gut to determine aggressiveness and length of therapeutic agents. Nutritional interventions of diet/supplement/ herbal combinations are well known to improve gut barrier, gut motility, and gut microbiome as well as natural antimicrobial/antifungal agents alone or in combination with antibiotics to address gut overgrowth and dysbiosis [21]. These must be paired with education for incorporation of lifestyle/diet/eating habits to promote a shift back to normal physiological processes with restoration of secretions, digestion, absorption, defecation, gut motility, gut lining integrity, and commensal microbiome diversity and abundance. The simplest way to restore the gut is to support intrinsic digestive processes of the parasympathetic rest and digest: appreciate and prepare your foods, sit and eat, chew, don't eat if your arguing or step away for a minute to breathe and calm down before eating. Using the process of the 4-Rs, Remove/Replace/Reinoculate/Repair $[1,15,20,48,52-57]$.

- Remove offending foods which cause symptomatic reactions, perform IgG food sensitivity test, consider intelligent eating strategies of removing sugars/carbs, gluten, and processed foods while replacing with plant-based fruit, vegetables, fibers, grains, nuts, seeds containing high amounts of antioxidants and essential nutrients

- Replace antacids with digestive enzymes

- Reinoculate with prebiotic fibers which feed the microbiome, while eating probiotic rich "living foods" such as kimchi, kombucha, yogurt, kefir, sauerkraut and supplementing with a probiotic of multiple commensal strains, look for ones with Saccharomyces Boullardi, and Bacillus Spore as well

- Repair the gut lining with removal of gluten foods (wheat, barley, rye, and baked goods from all purpose flour a source of wheat), supplement with agents that restore the mucus layer such as, $\mathrm{N}$-acetyl-glucosamine, DGL, colostrum.

Optimal health stems from gut-brain balance with physiological digestive function of secretions and motility, bowel movements, and symbiosis of the gut microbiome which is attained utilizing the 
body's intrinsic mechanisms of function and physiology. If treatment is required to rebalance, support the underlying physiological function and balance can be restored.

\section{Bibliography}

1. Guilliams T. "Functional Strategies for the Management of Gastrointestinal Disorders". Point Institute Wisconsin (2016).

2. Foster JA., et al. "Gut-brain axis: how the microbiome influences anxiety and depression". Trends in Neuroscience 36.5 (2013): 305-312.

3. Breton J., et al. "Ecotoxicology inside the gut: impact of heavy metals on the mouse microbiome". BMC Pharmacology and Toxicology 14 (2013): 62.

4. Miro-Blanch J and Yanes O. "Epigenetic Regulation at the Interplay Between Gut Microbiota and Host Metabolism". Frontier in Genetics (2019).

5. Hullar MA and Fu BC. "Diet, the gut microbiome, and epigenetics". Cancer Journal 20.3 (2014): 170-175.

6. Paul B., et al. "Influences of diet and the gut microbiome on epigenetic modulation in cancer and other diseases". Clinical Epigenetics 7 (2015): 112.

7. Quigley EM. "Microflora modulation of motility". Journal of Neurogastroenterology and Motility 17.2 (2011): 140-147.

8. Konturek PC., et al. "Stress and the gut: pathophysiology, clinical consequences, diagnostic approach and treatment options". Journal of Physiology and Pharmacology 62.6 (2011): 591-599.

9. Bruno G., et al. "Proton pump inhibitors and dysbiosis: Current knowledge and aspects to be clarified". World Journal of Gastroenterology 25.22 (2019): 2706-2719.

10. Kines K., et al. "Nutritional Interventions for Gastroesophageal Reflux, Irritable Bowel Syndrome, and Hypochlorhydria: A Case Report". Integratated Medicine (Encinitas) 15.4 (2016): 49-53.

11. Khayyatzadeh., et al. "Dietary behaviors in relation to prevalence of irritable bowel syndrome in adolescent girls". Journal of Gastroenterology and Hepatology 33.2 (2018): 404-410.
12. Gropper SS and Smith JL. "Advanced Nutrition and Human Metabolism". 6th ed. Wadsworth: Cengage Learning (2013).

13. Martinez KB., et al. "Western diets, gut dysbiosis, and metabolic diseases: Are they linked?" Gut Microbes 8.2 (2017): 130142.

14. El-Salhy M., et al. "Dietary fiber in irritable bowel syndrome (Review)". International Journal of Molecular Medicine 40.3 (2017): 607-613.

15. Hall John E. "Guyton and Hall Textbook of Medical Physiology". 13th ed. Guyton Physiology. London, England: W B Saunders (2015).

16. Browning KN and Travagli RA. "Central nervous system control of gastrointestinal motility and secretion and modulation of gastrointestinal functions". Comprehensive Physiology 4.4 (2014): 1339-1368.

17. Power ML and Schulkin J. "Anticipatory physiological regulation in feeding biology: cephalic phase responses". Appetite 50.2-3 (2008): 194-206.

18. Miquel-Kergoat S., et al. "Effects of chewing on appetite, food intake and gut hormones: A systematic review and meta-analysis". Physiology Behavior 151 (2015): 88-96.

19. Compare D., et al. "Effects of long-term PPI treatment on producing bowel symptoms and SIBO". European Journal of Clinical Investigation 41.4 (2011): 380-386.

20. Lacy BE., et al. "Pathophysiology, evaluation, and treatment of bloating: hope, hype, or hot air?". Gastroenterology and Hepatology (N Y) 7.11 (2011): 729-739.

21. Chedid V., et al. "Herbal therapy is equivalent to rifaximin for the treatment of small intestinal bacterial overgrowth". Global Advances in Health and Medicine 3.3 (2014): 16-24.

22. Gantzer J. "Eat Your Fats, Skip the Carbs: Here's Why. A Dietary Fat Metabolic Journey". Acta Scientific Neurology Special Issue 1 (2019): 01-04.

23. Green CL and Lamming DW. "Regulation of metabolic health by essential dietary amino acids". Mechanisms of Ageing and Development 177 (2019): 186-200. 
24. Ward E. "Addressing nutritional gaps with multivitamin and mineral supplements". Nutrition Journal 13 (2014): 72.

25. Huang D. "Dietary Antioxidants and Health Promotion". Antioxidants (Basel) 7.1 (2018): 9.

26. Lord RS and Bralley JA. "Laboratory Evaluations for Integrative and Functional Medicine". 2nd. Duluth, GA: Genova Diagnostics (2012).

27. Lord R. "Paths to Health: Gut Microbes". Chanhassen, MN: Arrowhead Bioscience (2018).

28. Jazani NH., et al. "Impact of Gut Dysbiosis on Neurohormonal Pathways in Chronic Kidney Disease”. Diseases 7.1 (2019): 21.

29. Guerreiro CS., et al. "Diet, Microbiota, and Gut PermeabilityThe Unknown Triad in Rheumatoid Arthritis". Frontiers in Medicine (Lausanne) 5 (2018): 349.

30. Wallace JL., et al. "Hydrogen sulfide: an agent of stability at the microbiome-mucosa interface". American Journal of Physiology-Gastrointestinal and Liver Physiology 314.2 (2018): G143G149.

31. Blachier F., et al. "Cysteine-derived hydrogen sulfide and gut health: a matter of endogenous or bacterial origin". Current Opinion in Clinical Nutrition and Metabolic Care 22.1 (2019): 68-75.

32. Barton LL., et al. "Sulfur Cycling and the Intestinal Microbiome”. Digestive Diseases and Sciences 62.9 (2017): 2241-2257.

33. Tang Y., et al. "Gut Microbiota Dysbiosis Enhances MigraineLike Pain Via TNF $\alpha$ Upregulation". Molecular Neurobiology (2019).

34. Lee SY., et al. "Microbiome in the Gut-Skin Axis in Atopic Dermatitis". Allergy, Asthma and Immunology Research 10.4 (2018): 354-362.

35. Clapp M., et al. "Gut microbiota's effect on mental health: The gut-brain axis". Clinical Practice 7.4 (2017): 987.

36. Rogers GB., et al. "From gut dysbiosis to altered brain function and mental illness: mechanisms and pathways". Molecular Psychiatry 21.6 (2016): 738-748.
37. Li Y., et al. "The Role of Microbiome in Insomnia, Circadian Disturbance and Depression". Frontiers in Psychiatry 9 (2018): 669.

38. Du Preez S., et al. "A systematic review of enteric dysbiosis in chronic fatigue syndrome/myalgic encephalomyelitis". Systematic Review 7.1 (2018): 241.

39. Sachdev AH and Pimentel M. "Gastrointestinal bacterial overgrowth: pathogenesis and clinical significance". Therapeutic Advances in Chronic Disease 4.5 (2013): 223-231.

40. Mohajeri MH., et al. "The role of the microbiome for human health: from basic science to clinical applications". European Journal of Nutrition 57 (2018): 1-14.

41. Imhann F., et al. "Proton pump inhibitors affect the gut microbiome”. Gut 65 (2016): 740-748.

42. Cater RE. "Helicobacter (aka Campylobacter) pylori as the major causal factor in chronic hypochlorhydria". Medical Hypotheses 39.4 (1992): 367-374.

43. Waldum HL., et al. "Helicobacter pylori and gastric acid: an intimate and reciprocal relationship". Therapeutic Advances in Gastroenterology 9.6 (2016): 836-844.

44. Lin HC. "Small intestinal bacterial overgrowth: a framework for understanding irritable bowel syndrome". JAMA 292 (2004): 852-858.

45. Esposito I., et al. "Breath test for differential diagnosis between small intestinal bacterial overgrowth and irritable bowel disease: an observation on non-absorbable antibiotics". World Journal of Gastroenterology 13.45 (2007): 6016-6021.

46. Anderson JM and Van Itallie CM. "Physiology and function of the tight junction". Cold Spring Harbor Perspectives in Biology 1.2 (2009): a002584.

47. $\mathrm{Mu}$ Q., et al. "Leaky Gut As a Danger Signal for Autoimmune Diseases". Frontiers in Immunology 8 (2017): 598.

48. Fasano A. "Zonulin, regulation of tight junctions, and autoimmune diseases". Annals of the New York Academy of Sciences 1258.1 (2012): 25-33. 
49. Hollon J., et al. "Effect of gliadin on permeability of intestinal biopsy explants from celiac disease patients and patients with non-celiac gluten sensitivity". Nutrients 7.3 (2015): 15651576.

50. Foster JA., et al. "Stress and the gut-brain axis: Regulation by the microbiome". Neurobiology Stress 7 (2017): 124-136.

51. Kelly JR., et al. "Breaking down the barriers: the gut microbiome, intestinal permeability and stress-related psychiatric disorders". Frontiers in Cell Neuroscience 9 (2015): 392.

52. Ianiro G., et al. "Digestive Enzyme Supplementation in Gastrointestinal Diseases". Current Drug Metabolism 17.2 (2016): 187-193.

53. Magge S., et al. "Low-FODMAP Diet for Treatment of Irritable Bowel Syndrome". Gastroenterology and Hepatology (N Y) 8.11 (2012): 739-745.

54. Tam., et al. "The Intestinal Life Cycle of Bacillus subtilis and Close Relatives". Journal of Bacteriology 188.7 (2006): 26922700.

55. Salvatore S., et al. "A pilot study of N-acetyl glucosamine, a nutritional substrate for glycosaminoglycan synthesis, in paediatric chronic inflammatory bowel disease". Aliment Pharmacology Therapy 14.12 (2000): 1567-1579.

56. Murray MT. “Glycyrrhiza glabra (Licorice)”. Textbook of Natural Medicine (2020): 641-647.e3.

57. Hałasa M., et al. “Oral Supplementation with Bovine Colostrum Decreases Intestinal Permeability and Stool Concentrations of Zonulin in Athletes". Nutrients 9.4 (2017): 370.

\section{Volume 4 Issue 11 November 2021}

(C) All rights are reserved by Jennifer Gantzer.

Citation: Jennifer Gantzer. "Supporting Gut Health by Homeostasis and Intrinsic Mechanisms". Acta Scientific Neurology 4.11 (2021): 10-17. 